


ISSN: 0067-2904

\title{
Synthesis, Molecular Structure from the X-ray Diffraction Data of the Powder $\left(1 E, 1^{\prime} E\right)$-1,1'-(1,4-Phenylene)Bis $(N$-(Adamantan-1- yl)methanimine)
}

\author{
Shukkur A. Hamed \\ Department of Chemistry, College of Education for Pure Science,University Of Anbar, Al-Anbar, Iraq \\ Received: 7/7/2020 \\ Accepted: 16/10/2020

\begin{abstract}
The title compound was synthesized by condensation between two equivalents adamantan-1-ylamine and one equivalent of benzene-1,4-dicarbaldehyde in $n-\mathrm{BuOH}$ and produced a good yield $87 \%$ of new bis Schiff base. The compound skeleton was affirmed by FTIR, ${ }^{1} \mathrm{H}$ NMR, LC-MS, and X-ray powder diffraction. The structure was solved by a parallel tempering process and refined by using Rietveld refinement. Two adamantan-1-ylimino groups are connected in the anti-positions to the planar central 1,4-dimethylbenzene group. All rings of the adamantyl group possess normal chair conformation.
\end{abstract}

Keywords: Powder X-ray diffraction, Adamantan-1-ylamine, Bis Schiff base.
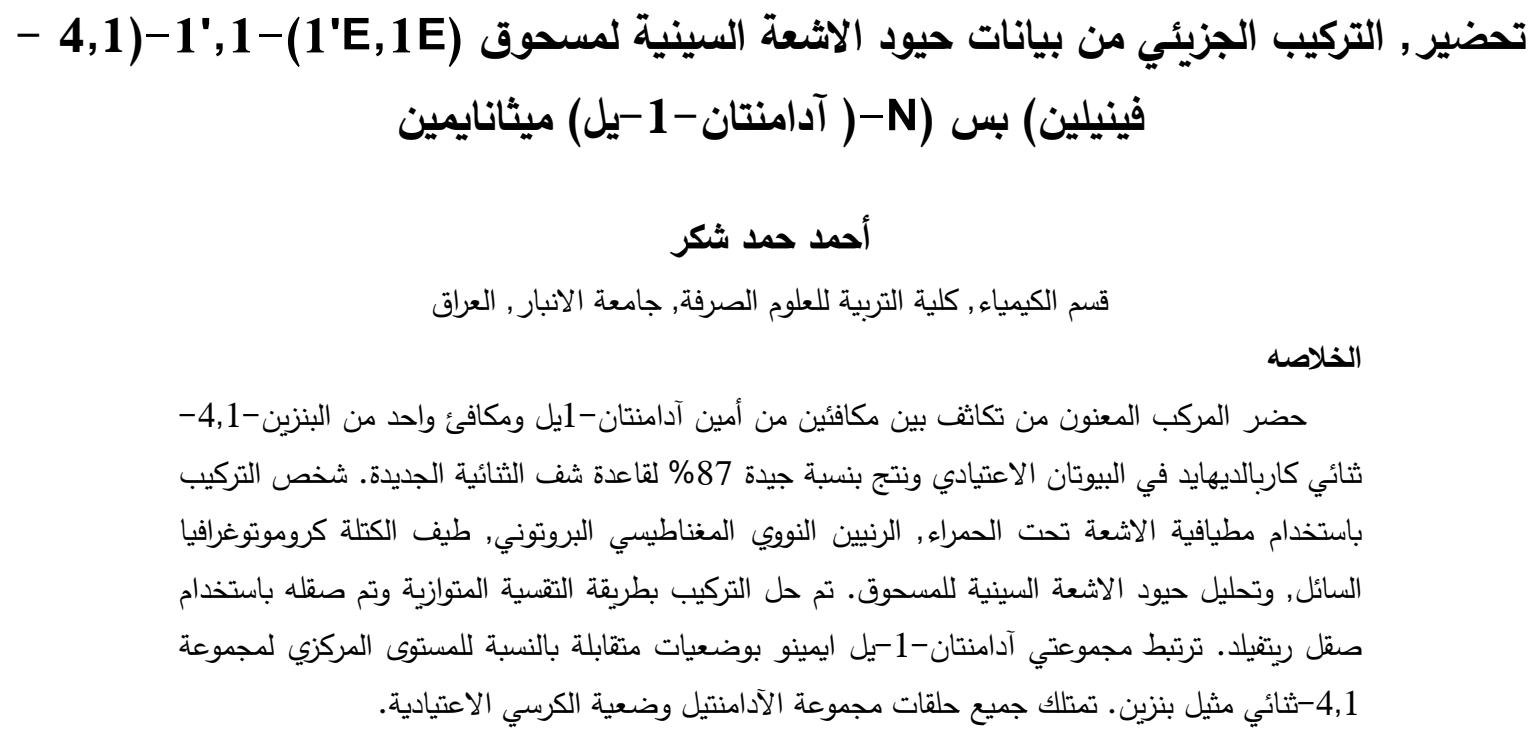

\section{Introduction}

The molecular design of adamantane and its derivatives are actual importance for researchers in molecular technology. Adamantan-1-ylamine is an alicyclic tricyclic amine which has an unusual skeleton adamantyl fragment consisting of three fused cyclohexane rings in a chair conformation [1]. It is the smallest repeating fragment of the diamond lattice [2]. The synthesis of molecules containing two or more pharmaceutical fragments in their structure allows for the development of new types of biological properties and for the extension of known curative effects. Adamantan-1-amine and its derivatives have been a good source for a wide scope of pharmaceutical drugs. In particular, they have 
been effective in the development of drugs for Parkinson's disease [3], treatment and protection against influenza virus (A) [4], anti-microbial drugs [5], and anti-inflammatory drugs [6]. Furthermore, compounds that contain azomethines are reactive intermediates for organic synthesis in different fields and have biological [7, 8], anti-viral [9], anti-bacterial [10,11], and anti-fungal [12] effects, as well as inhibiting anti-microbial agents [13]. A search in the CCDC Database survey gave few results containing adamantan-1-ylaminomethylbenzene fragment or 1,4-di(aminomethyl)benzene fragment [14-20]. Structures similar to the title compound are absent in the database. This work presents new applications of bis imine $\left(1 E, 1^{\prime} E\right)-1,1^{\prime}-(1,4$-phenylene) bis ( $N$-(adamantan-1yl)methanimine) as a predece-ssor for the synthesis of new compounds. The structure of the desired compound was confirmed by the use of the X-ray powder diffraction technique.

\section{Materials and Methods}

The materials were purchased from Merck (Germany) and Romil Co. (UK). The melting point was measured by the Stuart SMP-10 apparatus. FTIR, ${ }^{1} \mathrm{H}$ NMR and LC-MS spectra were recorded on Bruker Tensor 27, Bruker UltraShield (300 MHz, CDCl3), and Thermo Scientific Exactive Plus Orbitrap LC-MS spectrometer, respectively. X-ray powder diffraction pattern was measured on the PANalytical Empyrean diffractometer.

\section{Synthesis of the title compound}

To a hot solution of adamantan-1-ylamine $(4 \mathrm{~g}, 0.026 \mathrm{~mol})$ in $15 \mathrm{ml} n$-BuOH a solution of benzene1,4-dicarbaldehyde $(1.74 \mathrm{~g}, 0.013 \mathrm{~mol})$ in $10 \mathrm{ml} n$-BuOH was added. The mixture was refluxed with stirring for $30 \mathrm{~min}$ and was then left for $3 \mathrm{~h}$. The crude product was filtered and evaporated. The product was collected and re-crystallized from $\mathrm{EtOH}$ or $i$-PrOH.

The properties and spectral data of the title compound:

White needles, yield $4.6 \mathrm{~g}(87 \%)$, m.p $243-244^{\circ} \mathrm{C}$. FTIR, $v, \mathrm{~cm}^{-1}: 1633(\mathrm{C}=\mathrm{N}), 3013(\mathrm{C}-\mathrm{H}$ aromatic), 2904, 2846 (C- $\mathrm{H}$ aliphatic). ${ }^{1} \mathrm{H} \mathrm{NMR}\left(\mathrm{CDCl}_{3}, \delta, 300 \mathrm{MHz}\right.$, p.p.m.): (adamantyl group $1.66\left(\mathrm{~s}, 12 \mathrm{H}, 6 \mathrm{CH}_{2}\right), 1.85(\mathrm{~m}, 6 \mathrm{H}, 6 \mathrm{CH}), 2.23$ (broad s, $\left.\left.12 \mathrm{H}, 6 \mathrm{CH}_{2}\right)\right), 7.92-8.26(\mathrm{~m}, 4 \mathrm{H}$, Ar-ring), 8.32 (s, 2H, $2 \mathrm{CH}=\mathrm{N})$. LC-MS, m/z (\%): $400.89[M]+(100)$.

$\mathrm{X}$-ray powder structure of the title compound: The powder as very small needles was obtained by allowing the saturated EtOH solution of the compound to stand for four days. The details of crystallographic data are shown in Tables 1-4.

\section{Results and Discussion}

The route of the synthesis of the title compound is shown in scheme 1 , which includes the synthesis of new bis Schiff base $\left(1 E, 1^{\prime} E\right)-1,1^{\prime}-(1,4-$ phenylene)bis( $N$-(adamantan-1-yl)methanimine) by condensation between two equivalents adamantan-1-ylamine and one equivalent of benzene-1,4dicarbaldehyde in the presence of $n$-butanol as a solvent, and produced a good yield (87\%). The skeleton of the compound was affirmed by FTIR, ${ }^{1} \mathrm{H}$ NMR, LC-MS, and powder X-ray diffraction.

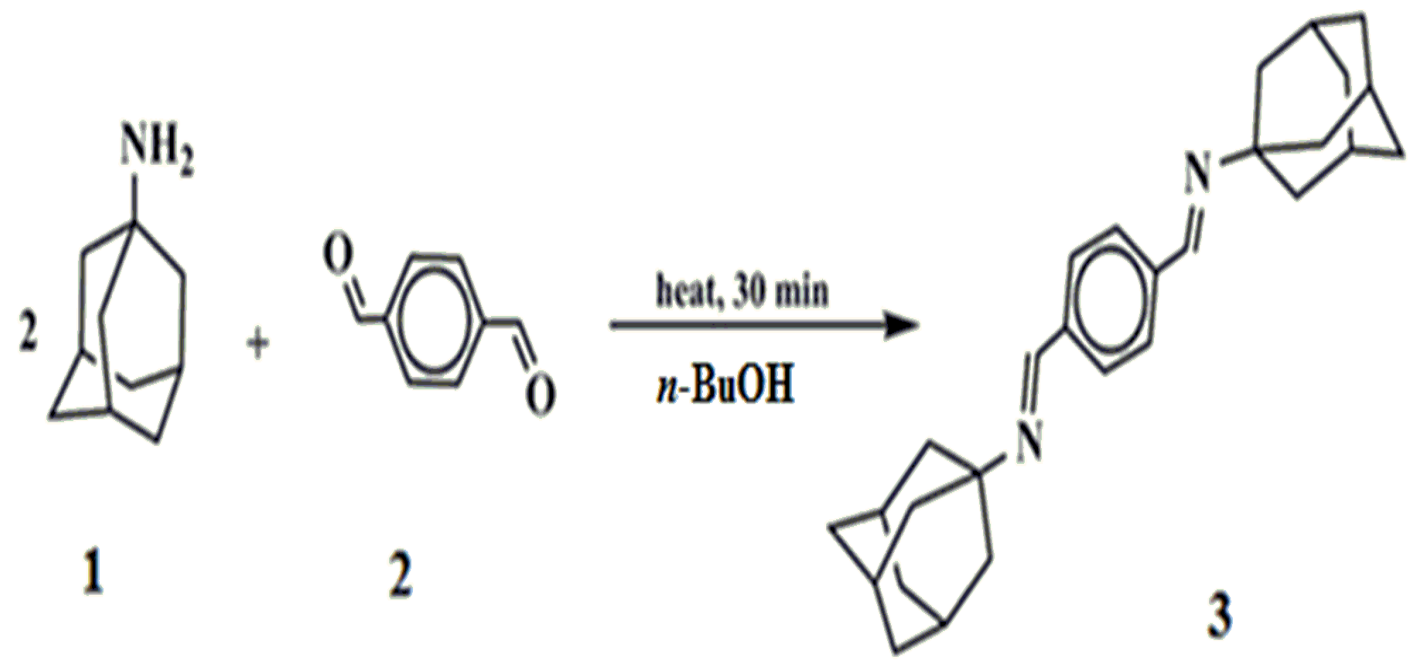

Scheme 1- For the synthesis of the title structure 
FTIR spectrum displayed evanescence in the stretching vibration bands of groups $\left(-\mathrm{NH}_{2}\right)$ and $(\mathrm{C}=\mathrm{O})$ for amine and benzene-1,4-dicarbaldehyde respectively with appeared stretching vibration band of the azomethine group $(\mathrm{C}=\mathrm{N})$ at $1633 \mathrm{~cm}^{-1}$. The stretching vibration band for $\left(\mathrm{C}-\mathrm{H}_{\text {aromatic }}\right)$ appeared at $3013 \mathrm{~cm}^{-1}$, and stretching vibration bands for $\left(\mathrm{C}-\mathrm{H}_{\text {aliphatic }}\right)$ appeared at 2846 and $2904 \mathrm{~cm}^{-1}$ (Figure1). The ${ }^{1} \mathrm{H}$ NMR spectrum for $(2 \mathrm{CH}=\mathrm{N})$ protons displayed the presence of a down-field singlet at $\delta$ $8.32 \mathrm{ppm}$ and protons of phenyl group displayed two singlets at $\delta 7.92$ and $8.26 \mathrm{ppm}$. The adamantyl group exhibited signals as a singlet, multiplet, and broad singlet at $\delta 1.66\left(12 \mathrm{H}, 6 \mathrm{CH}_{2} \mathrm{C}-\mathrm{N}\right), 1.85(6 \mathrm{H}$, $6 \mathrm{CH})$ and $2.23\left(12 \mathrm{H}, 6 \mathrm{CH}_{2}\right) \mathrm{ppm}$, respectively (Figure-2). Moreover, LC-mass spectrum was identical to its molecular weight, $\mathrm{m} / \mathrm{z}(\%): 400.89[\mathrm{M}]+(100)$ (Figure-3). The formation of the bis imine derivative title compound was occurred according to a similar suggested mechanism in literature [12] but in the absent catalyst. The two pairs of electrons nitrogen of two molecules amine attacks to the dicarbonyl group by nucleophilic addition produced dihemi-aminal and then the left two water molecules and gave the target compound. Scheme- 2 .
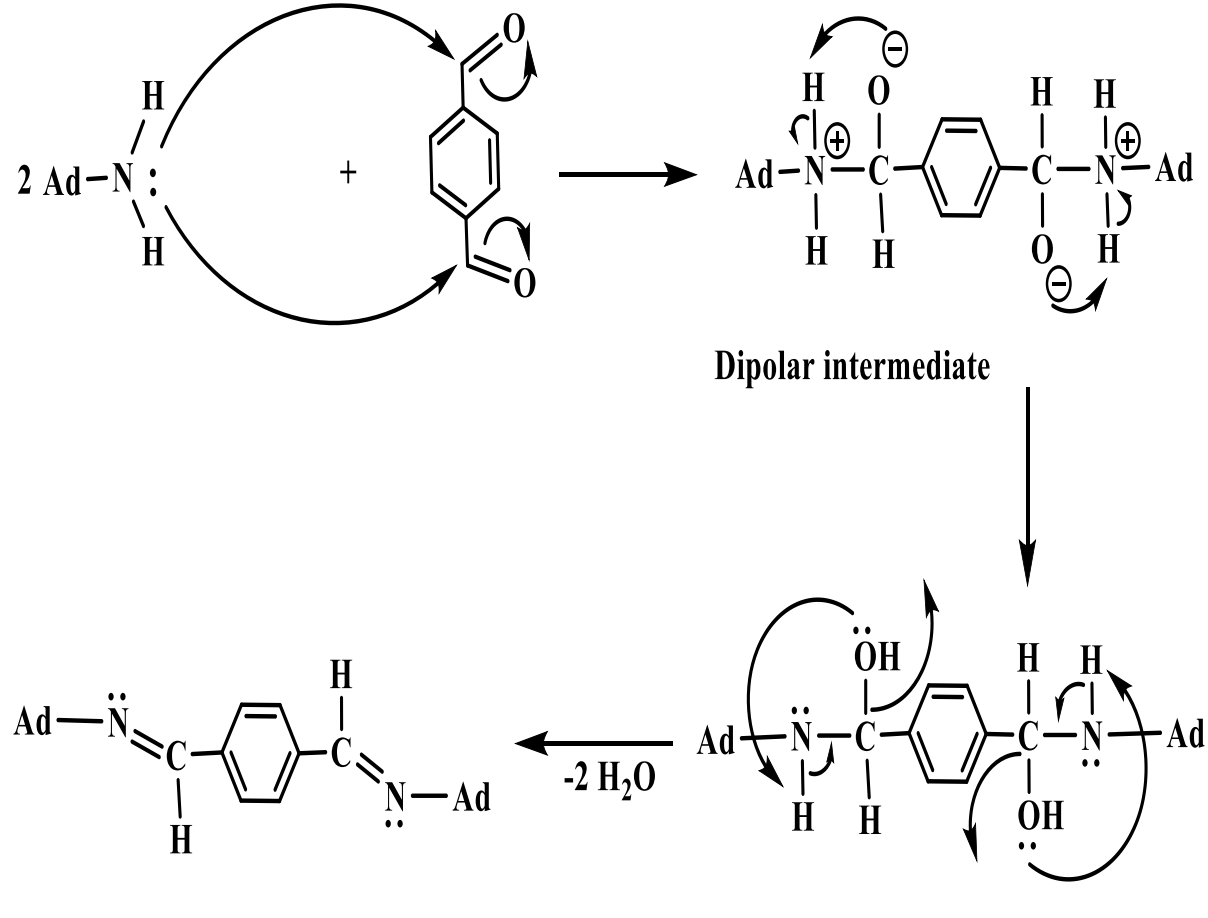

Bis imine

Bis carbinol amine

\section{$\mathrm{Ad}=$ adamantyl group}

Scheme 2-The suggested mechanism of bis imine for target compound 


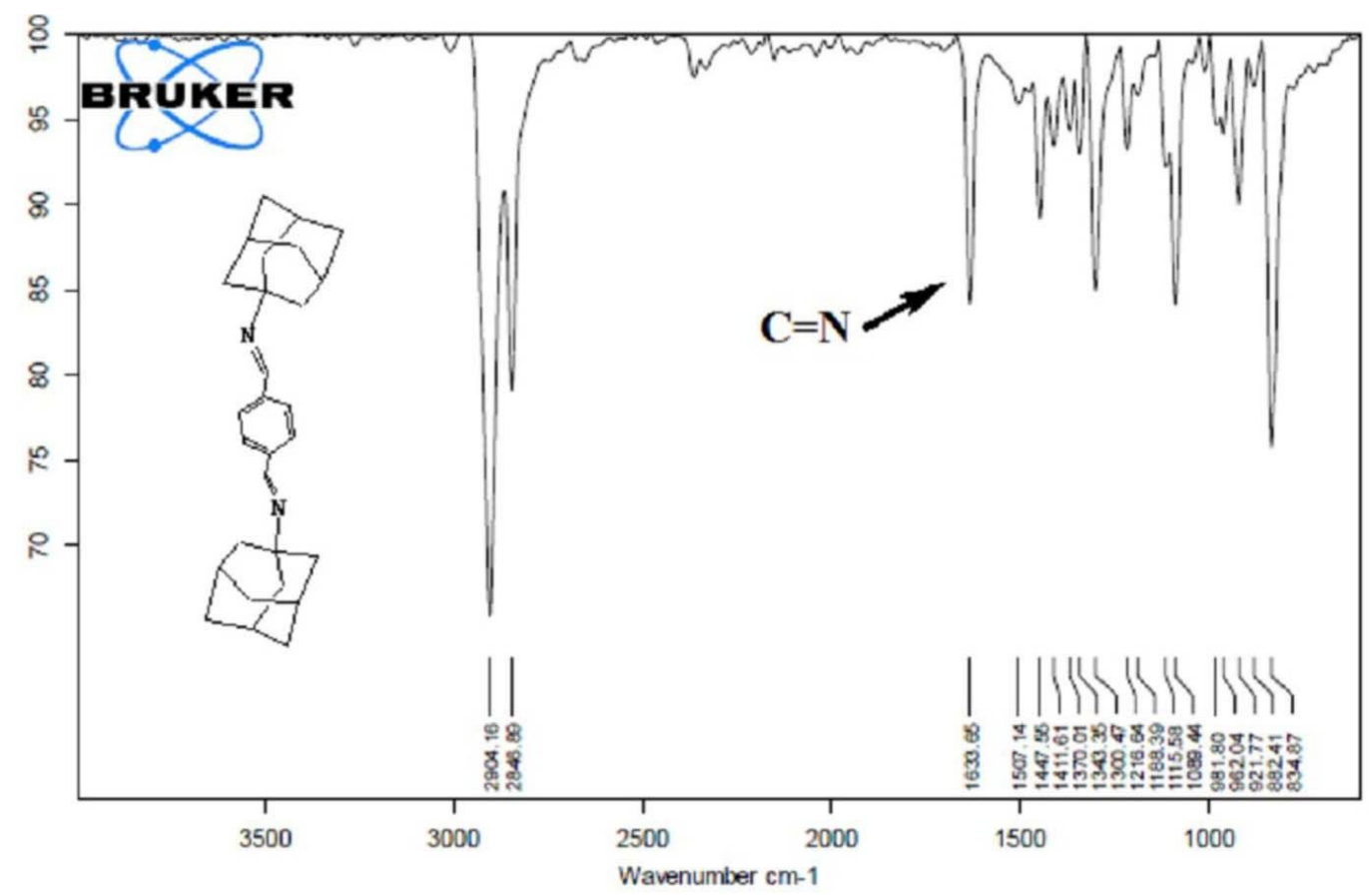

Figure 1-FTIR spectrum of the title compound

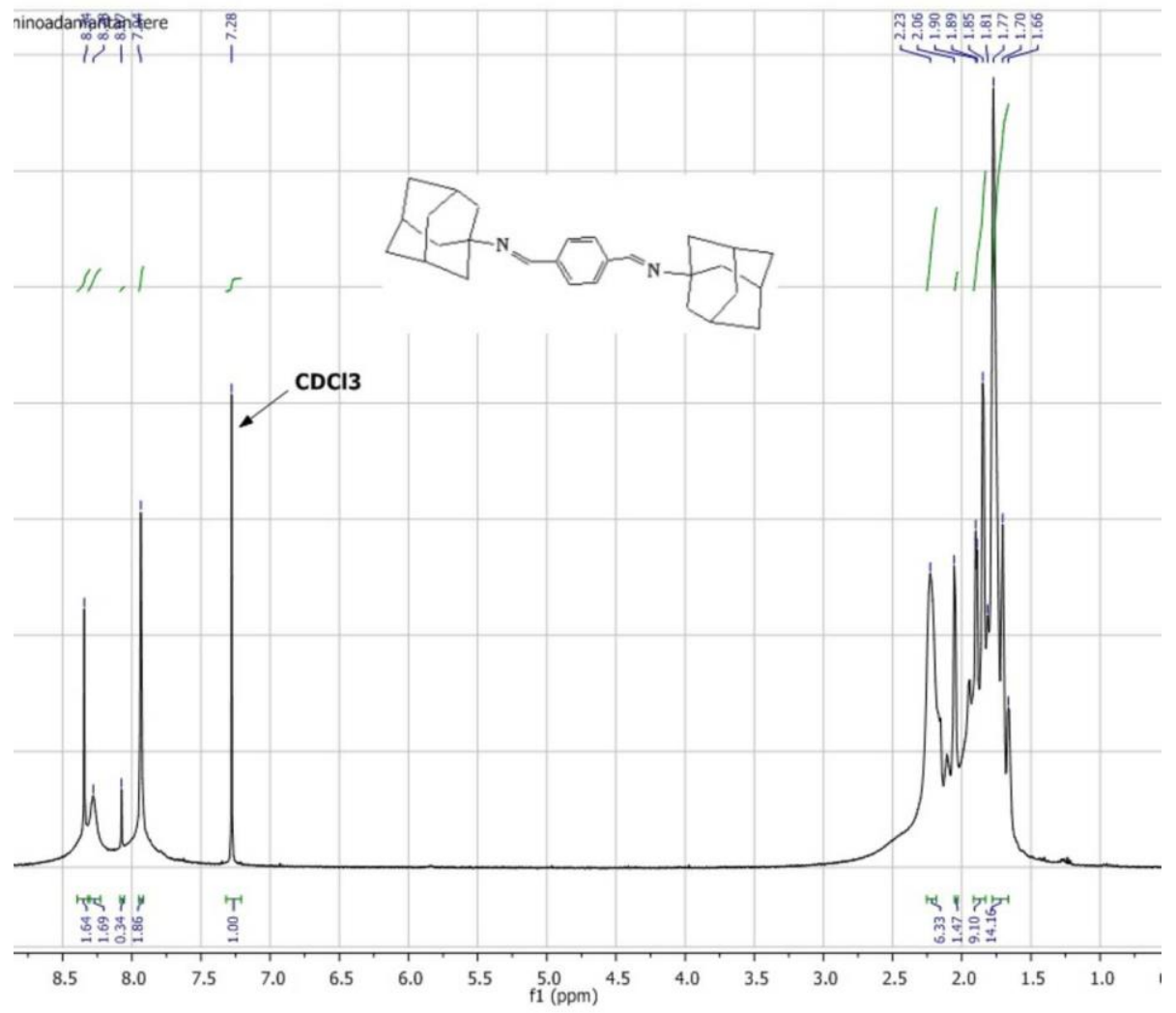

Figure 2- ${ }^{1} \mathrm{H}$ NMR spectrum of the title compound 


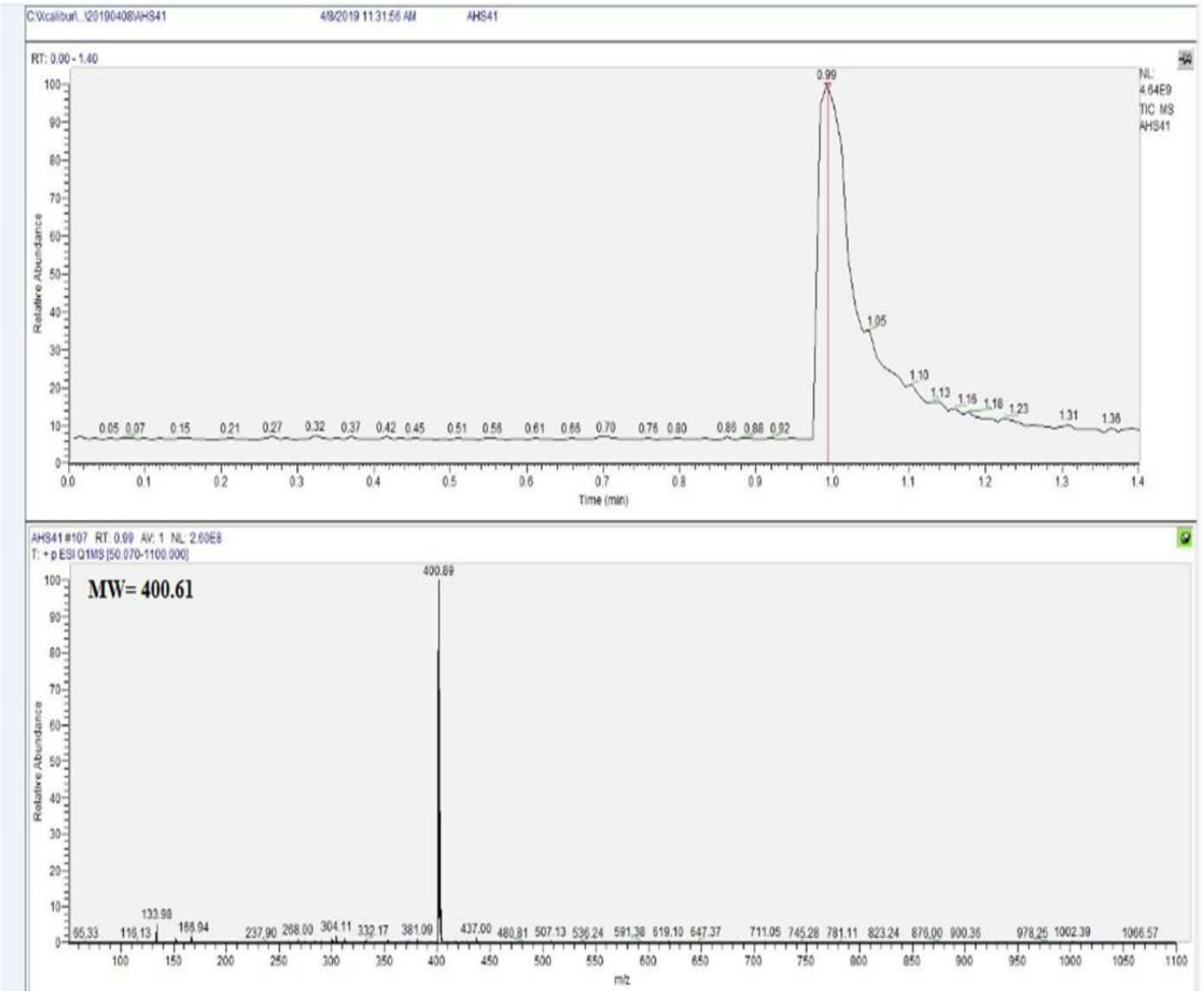

Figure 3-LC-MS spectrum of the title compound

\section{Crystallographic Study}

The molecular structure of the title compound was displayed in Figure- 4. Its crystalline data were as follows: $\mathrm{C}_{28} \mathrm{H}_{36} \mathrm{~N}_{2}, M . W=400.59$ g.mol ${ }^{-1}$, system with space group: Monoclinic, $C 2 / c, \mathrm{a}=28.249$ (10) $\mathrm{A}^{\circ}, \mathrm{b}=6.450(18) \mathrm{A}^{\circ}, \mathrm{c}=12.509$ (4) $\mathrm{A}^{\circ}, \beta^{\circ}=91.88$ (4), $V=2278$ (6) $\mathrm{A}^{\circ 3}, \mathrm{Z}=4$, Rad. type $\mathrm{Cu} K \alpha, \lambda$ $=1.5418 \mathrm{~A}^{\circ}$, Specimen shape, size: Flat sheet $(15 \times 1) \mathrm{mm}$.

The X-ray powder diffraction pattern in (Figure-6) was measured by computer programs in the $\theta$ range $5-70^{\circ}$ on PANalytical Empyrean diffractometer [21]. The structure was solved by parallel tempering technique using FOX [22], and refined by Rietveld refinement procedure was made by MRIA [23] with constrained isotropic thermal parameters for all non-hydrogen atoms. Molecular graphics was made by Mercury [24]. Hydrogen atoms were calculated and not refined. The bond length of $(\mathrm{N} 6-\mathrm{C} 12)$ for $(\mathrm{N}=\mathrm{C})$ is normal $1.32(2) \mathrm{A}^{\circ}$. In the crystal structure of the target compound, was observed two adamantan-1-ylimino groups are arranged in the anti-positions to the planar central 1, 4-dimethylbenzene group (Figure-4). The molecule is located on the inversion center. All rings of adamantyl moiety possess normal chair conformation. The details of the crystal data are provided in Tables-(1-4). 


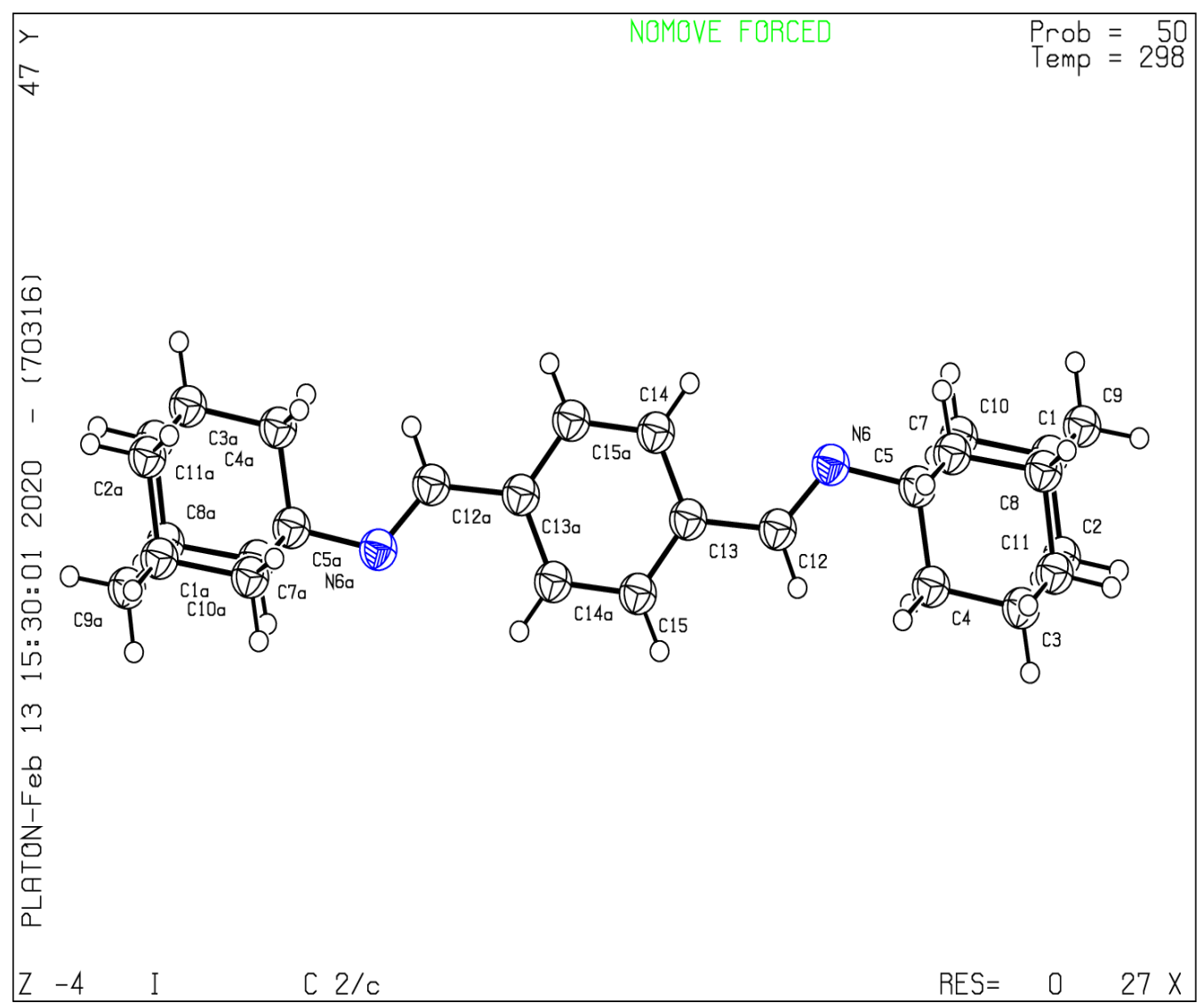

Figure 4-Data block: Showing the molecular structure, atoms numbering and displacement ellipsoids are drawn at $50 \%$ probability level of the title compound

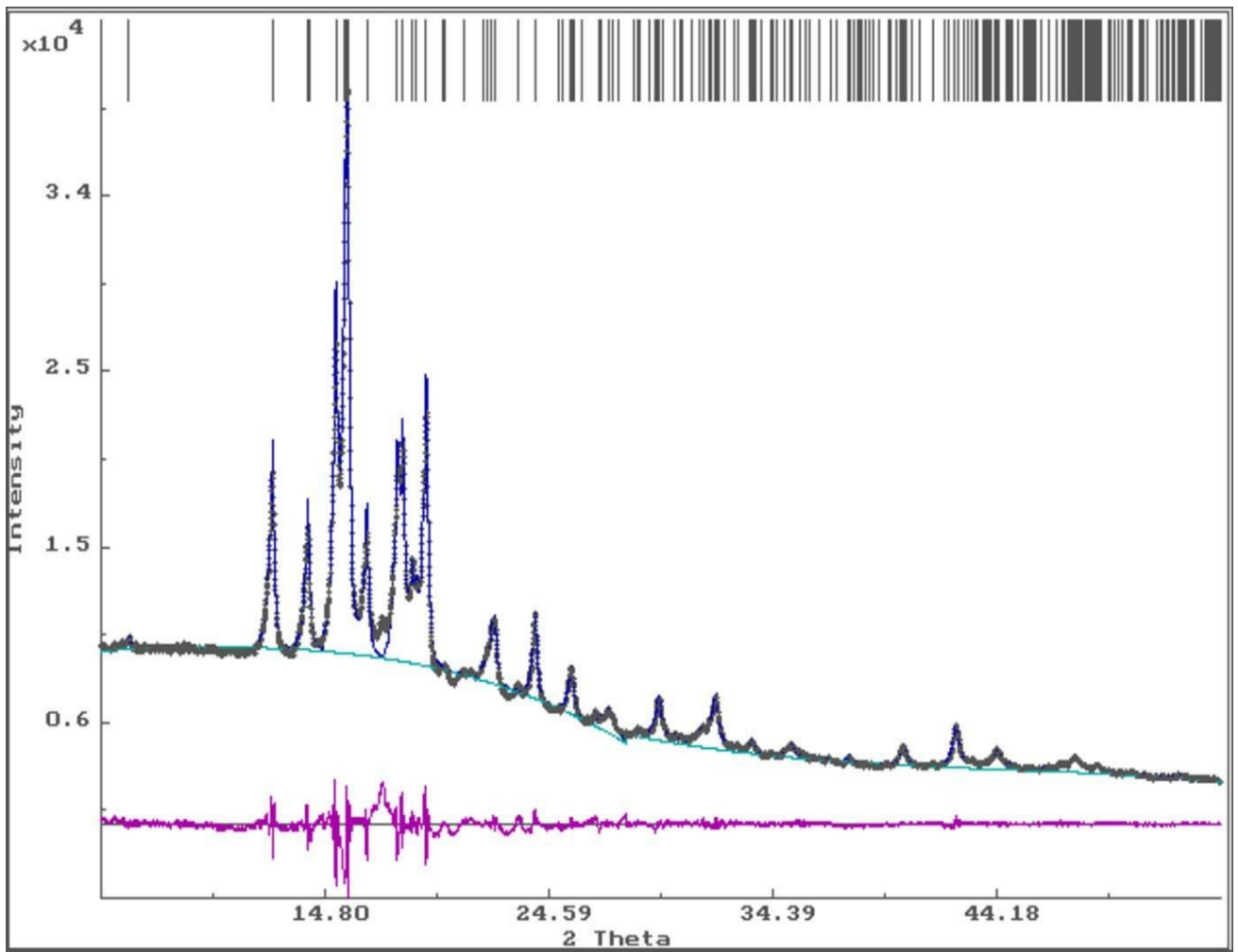

Figure 5-Powder diffraction pattern of the title compound 
Table 1-Crystal data and refinement details of the title compound

\begin{tabular}{|c|c|}
\hline Crystal data & \\
\hline$M . F$ & $\mathrm{C}_{28} \mathrm{H}_{36} \mathrm{~N}_{2}$ \\
\hline$M . W$ & 400.59 \\
\hline $\begin{array}{l}\text { Crystal system and } \\
\text { group of space }\end{array}$ & Monoclinic, $C 2 / c$ \\
\hline Temp. & $298 \mathrm{~K}$ \\
\hline$a, b, c\left(\mathrm{~A}^{\circ}\right)$ & $28.249(10), 6.450(18), 12.509(4)$ \\
\hline$\beta\left(^{\circ}\right)$ & $91.88(4)$ \\
\hline$V\left(\mathrm{~A}^{\mathrm{o3}}\right)$ & $2278(6)$ \\
\hline$Z$ & 4 \\
\hline Type of radiation & $\mathrm{Cu} K \alpha, \lambda=1.5418 \mathrm{~A}^{\circ}$ \\
\hline Shape and size of specimen & Flat sheet, $15 \times 1 \mathrm{~mm}$ \\
\hline \multicolumn{2}{|l|}{ Collection data } \\
\hline Diffractometer & EMPYREAN (PANanalytical, 2011) \\
\hline Mounting of specimen & Thin layer over the non-diffracting silicon plate \\
\hline Mode collection data & Reflection \\
\hline Scan process & Persistent \\
\hline $2 \theta$ values & $2 \theta_{\min }=5.00^{\circ}, 2 \theta_{\max }=70.00^{\circ}, 2 \theta_{\text {step }}=0.01^{\circ}$ \\
\hline \multicolumn{2}{|l|}{ Refinement } \\
\hline R-factors and quality of fit & $\begin{array}{c}\mathrm{R}_{\mathrm{p}}=0.026, \mathrm{R}_{\mathrm{wp}}=0.033, \mathrm{R}_{\text {exp }}=0.013 \\
\mathrm{R}_{\text {Bragg }}=0.109^{\circ}, \chi 2=7.618\end{array}$ \\
\hline Parameters number & 104 \\
\hline Restraints number & 48 \\
\hline Treatment of H-atoms & Parameters of $\mathrm{H}$-atoms not refined \\
\hline Code of symmetry & (i) $)^{-x,-y+1,-z+1}$ \\
\hline $\begin{array}{l}\text { CCDC reference, } \\
\text { deposition number }\end{array}$ & 1971146 \\
\hline
\end{tabular}

Table 2-Geometric parameters (Bonds lengths) $\left(A,{ }^{\circ}\right)$ of the title compound

\begin{tabular}{|c|c|c|c|}
\hline Bond & $d\left(A,^{o}\right)$ & Bond & $d\left(A,^{o}\right)$ \\
\hline C1-C2 & $1.534(19)$ & C1-H1 & 0.9800 \\
\hline C1-C9 & $1.57(3)$ & C2-H2A & 0.9700 \\
\hline C1-C10 & $1.54(3)$ & C2-H2B & 0.9700 \\
\hline C2-C3 & $1.55(2)$ & C3-H3 & 0.9800 \\
\hline C3-C4 & $1.535(19)$ & C4-H4A & 0.9700 \\
\hline C3-C11 & $1.54(2)$ & C4-H4B & 0.9700 \\
\hline C4-C5 & $1.528(16)$ & C7-H7A & 0.9700 \\
\hline C5-N6 & $1.48(2)$ & C7-H7B & 0.9700 \\
\hline C5-C7 & $1.54(2)$ & C8-H8 & 0.9800 \\
\hline C5-C10 & $1.55(2)$ & C9-H9A & 0.9700 \\
\hline N6-C12 & $1.32(2)$ & C9-H9B & 0.9700 \\
\hline C7-C8 & $1.541(19)$ & C10-H10A & 0.9700 \\
\hline C8-C9 & $1.555(19)$ & C10-H10B & 0.9700 \\
\hline C8-C11 & $1.555(17)$ & C11-H11A & 0.9700 \\
\hline C12-C13 & $1.501(18)$ & C11-H11B & 0.9700 \\
\hline C13-C14 & $1.41(3)$ & C12-H12 & 0.9300 \\
\hline C13-C15 & $1.39(2)$ & C14-H14 & 0.9300 \\
\hline C14-C15i & $1.4213(2)$ & C15-H15 & 0.9300 \\
\hline C15-C14i & $1.4213(2)$ & & \\
\hline
\end{tabular}


Table 3- Geometric parameters (Bonds angles $\omega,{ }^{\circ}$ ) of the title compound

\begin{tabular}{|c|c|c|c|}
\hline Angle & $\omega,^{\circ}$ & Angle & $\omega,^{\circ}$ \\
\hline C2-C1-C9 & $106.5(7)$ & C11-C3-H3 & 110.5 \\
\hline $\mathrm{C} 2-\mathrm{C} 1-\mathrm{C} 10$ & $108.1(5)$ & C2-C3-H3 & 110.5 \\
\hline C9-C1-C10 & $104.9(4)$ & C5-C4-H4A & 109.7 \\
\hline $\mathrm{C} 1-\mathrm{C} 2-\mathrm{C} 3$ & $112.5(3)$ & C3-C4-H4A & 109.7 \\
\hline $\mathrm{C} 2-\mathrm{C} 3-\mathrm{C} 4$ & $107.1(3)$ & C5-C4-H4B & 109.7 \\
\hline C2-C3-C11 & $109.7(5)$ & C3-C4-H4B & 109.7 \\
\hline C4-C3-C11 & $108.6(3)$ & H4A-C4-H4B & 108.2 \\
\hline C3-C4-C5 & $110.0(5)$ & C5-C7-H7A & 109.3 \\
\hline C4-C5-N6 & $111.0(9)$ & C8-C7-H7A & 109.3 \\
\hline C4-C5-C7 & $111.9(6)$ & C5-C7-H7B & 109.3 \\
\hline C4-C5-C10 & $107.4(4)$ & C8-C7-H7B & 109.3 \\
\hline N6-C5-C7 & $108.4(8)$ & H7A-C7-H7B & 108.0 \\
\hline N6-C5-C10 & $108.0(6)$ & $\mathrm{C} 7-\mathrm{C} 8-\mathrm{H} 8$ & 112.9 \\
\hline C7-C5-C10 & $109.9(2)$ & C9-C8-H8 & 112.9 \\
\hline C5-N6-C12 & $119.4(2)$ & C11-C8-H8 & 112.9 \\
\hline C5-C7-C8 & $111.6(8)$ & C8-C9-H9A & 108.0 \\
\hline C7-C8-C9 & $106.8(4)$ & C1-C9-H9A & 108.0 \\
\hline C7-C8-C11 & $106.0(3)$ & C8-C9-H9B & 108.0 \\
\hline C9-C8-C11 & $104.9(5)$ & C1-C9-H9B & 108.0 \\
\hline C1-C9-C8 & $116.9(4)$ & H9A-C9-H9B & 107.3 \\
\hline C1-C10-C5 & $111.9(5)$ & $\mathrm{C} 1-\mathrm{C} 10-\mathrm{H} 10 \mathrm{~A}$ & 109.2 \\
\hline C3-C11-C8 & $114.5(6)$ & C5-C10-H10A & 109.2 \\
\hline N6-C12-C13 & $126.3(4)$ & C1-C10-H10B & 109.2 \\
\hline C12-C13-C14 & $118.3(5)$ & C5-C10-H10B & 109.2 \\
\hline C12-C13-C15 & $121.0(5)$ & H10A-C10-H10B & 107.9 \\
\hline C14-C13-C15 & $120.6(8)$ & C3-C11-H11A & 108.6 \\
\hline $\mathrm{C} 13-\mathrm{C} 14-\mathrm{C} 15 \mathrm{i}$ & $119.6(7)$ & $\mathrm{C} 8-\mathrm{C} 11-\mathrm{H} 11 \mathrm{~A}$ & 108.6 \\
\hline C13-C15-C14i & $119.8(7)$ & C3-C11-H11B & 108.6 \\
\hline $\mathrm{C} 2-\mathrm{C} 1-\mathrm{H} 1$ & 112.3 & C8-C11-H11B & 108.6 \\
\hline $\mathrm{C} 10-\mathrm{C} 1-\mathrm{H} 1$ & 112.3 & H11A-C11-H11B & 107.6 \\
\hline C9-C1-H1 & 112.3 & N6-C12-H12 & 116.8 \\
\hline $\mathrm{C} 1-\mathrm{C} 2-\mathrm{H} 2 \mathrm{~A}$ & 109.1 & $\mathrm{C} 13-\mathrm{C} 12-\mathrm{H} 12$ & 116.8 \\
\hline C3-C2-H2A & 109.1 & C13-C14-H14 & 120.2 \\
\hline C1-C2-H2B & 109.1 & C15i-C14-H14 & 120.2 \\
\hline $\mathrm{C} 3-\mathrm{C} 2-\mathrm{H} 2 \mathrm{~B}$ & 109.1 & C13-C15-H15 & 120.1 \\
\hline $\mathrm{H} 2 \mathrm{~A}-\mathrm{C} 2-\mathrm{H} 2 \mathrm{~B}$ & 107.8 & C14i-C15-H15 & 120.1 \\
\hline $\mathrm{C} 4-\mathrm{C} 3-\mathrm{H} 3$ & 110.5 & & \\
\hline
\end{tabular}


Table 4-Parameters of displacement $\left(\mathrm{A}^{\circ 2}\right)$ for fractional atomic coordinates and isotropic or equivalent isotropic of the title compound

\begin{tabular}{|c|c|c|c|c|}
\hline atoms & $x$ & $y$ & $z$ & Uiso $^{*} / \mathrm{Ueq}_{\mathrm{q}}$ \\
\hline $\mathrm{C} 1$ & $0.1425(5)$ & $0.969(3)$ & $0.0600(13)$ & $0.05066^{*}$ \\
\hline $\mathrm{C} 2$ & $0.1416(5)$ & $0.766(2)$ & $-0.0039(8)$ & $0.05066^{*}$ \\
\hline $\mathrm{C} 3$ & $0.1552(5)$ & $0.575(3)$ & $0.0662(13)$ & $0.05066^{*}$ \\
\hline $\mathrm{C} 4$ & $0.1191(4)$ & $0.5625(16)$ & $0.1551(11)$ & $0.05066^{*}$ \\
\hline $\mathrm{C} 5$ & $0.1226(4)$ & 0.7559 (19) & $0.2255(10)$ & $0.05066^{*}$ \\
\hline N6 & $0.0886(4)$ & $0.745(2)$ & $0.3131(8)$ & $0.05066^{*}$ \\
\hline $\mathrm{C} 7$ & $0.1726(4)$ & $0.785(2)$ & $0.2750(9)$ & $0.05066^{*}$ \\
\hline $\mathrm{C} 8$ & $0.2099(6)$ & $0.806(2)$ & $0.1884(13)$ & $0.05066^{*}$ \\
\hline C9 & $0.1935(4)$ & 0.9865 (18) & $0.1138(13)$ & $0.05066^{*}$ \\
\hline $\mathrm{C} 10$ & $0.1091(4)$ & $0.9450(17)$ & $0.1539(10)$ & $0.05066^{*}$ \\
\hline C11 & $0.2049(4)$ & 0.6075 (19) & $0.1181(13)$ & $0.05066^{*}$ \\
\hline $\mathrm{C} 12$ & $0.0661(3)$ & $0.571(2)$ & $0.3308(8)$ & $0.05066^{*}$ \\
\hline $\mathrm{C} 13$ & $0.0311(4)$ & 0.5325 (19) & $0.4162(9)$ & $0.05066^{*}$ \\
\hline $\mathrm{C} 14$ & $0.0207(5)$ & $0.6963(12)$ & $0.4860(10)$ & $0.05066^{*}$ \\
\hline $\mathrm{C} 15$ & $0.0110(4)$ & $0.3373(14)$ & $0.4298(8)$ & $0.05066^{*}$ \\
\hline $\mathrm{H} 1$ & 0.1345 & 1.0894 & 0.0152 & $0.061 *$ \\
\hline $\mathrm{H} 2 \mathrm{~A}$ & 0.1635 & 0.7769 & -0.0618 & $0.061^{*}$ \\
\hline $\mathrm{H} 2 \mathrm{~B}$ & 0.1101 & 0.7455 & -0.0353 & $0.061^{*}$ \\
\hline $\mathrm{H} 3$ & 0.1544 & 0.4481 & 0.0231 & $0.061^{*}$ \\
\hline $\mathrm{H} 4 \mathrm{~A}$ & 0.0874 & 0.5513 & 0.1236 & $0.061 *$ \\
\hline H4B & 0.1252 & 0.4400 & 0.1984 & $0.061 *$ \\
\hline $\mathrm{H} 7 \mathrm{~A}$ & 0.1805 & 0.6671 & 0.3203 & $0.061^{*}$ \\
\hline H7B & 0.1732 & 0.9083 & 0.3194 & $0.061 *$ \\
\hline $\mathrm{H} 8$ & 0.2421 & 0.8256 & 0.2184 & $0.061^{*}$ \\
\hline H9A & 0.2161 & 0.9992 & 0.0574 & $0.061^{*}$ \\
\hline H9B & 0.1950 & 1.1139 & 0.1549 & $0.061^{*}$ \\
\hline H10A & 0.0769 & 0.9282 & 0.1261 & $0.061 *$ \\
\hline H10B & 0.1103 & 1.0700 & 0.1970 & $0.061 *$ \\
\hline H11A & 0.2277 & 0.6146 & 0.0620 & $0.061 *$ \\
\hline H11B & 0.2128 & 0.4877 & 0.1621 & $0.061 *$ \\
\hline H12 & 0.0725 & 0.4599 & 0.2859 & $0.061^{*}$ \\
\hline H14 & 0.0345 & 0.8258 & 0.4771 & $0.061 *$ \\
\hline H15 & 0.0183 & 0.2293 & 0.3839 & $0.061^{*}$ \\
\hline
\end{tabular}

\section{Conclusion}

The research included a synthesis of new bis Schiff base which contains adamantyl moiety by condensation between two equivalents adamantan-1-ylamine and one equivalent of benzene-1,4dicarbaldehyde in $n$-BuOH gave $\left(1 E, 1^{\prime} E\right)-1,1^{\prime}-(1,4$-phenylene) $b i s(N$-(adamantan-1-yl)methanimine) with good yield. The skeleton of the compound was affirmed by FTIR, ${ }^{1} \mathrm{H}$ NMR, LC-MS spectroscopy, and X-ray powder diffraction techniques. The structure was solved by a parallel tempering process and refined by Rietveld refinement. The positions of two adamantan-1-ylimino groups are connected in the anti-conformation to the planar central 1,4-dimethylbenzene group. The rings of the adamantyl fragment showed that have a normal chair conformation.

\section{Acknowledgement}

The author thanks Dr. Dmitry V. A. Department of Chemistry, Moscow State University, for support in performing the $\mathrm{X}$-ray crystallography analysis. 


\section{References}

1. Fort, R. C. 1976. Adamantane: The Chemistry of Diamond Molecules. Studies in Organic Chemistry. 5, Marcel Dekker, New York, USA.

2. Fort, R. C. and Schleyer P.von R. 1964. Adamantane: consequences of the Diamondoid Structure. Chem. Rev., 64: 277-300.

3. Davies, W. L., Grunert, R. R., Haff, R. F., Mcgahen, J. W., Neumayer, E. M., Paulshock, M., Watts J. C., Wood, T. R., Hermann, E. C. and Hoffmann, C. E. 1964. Antiviral Activity of 1Adamantanamine (Amantadine). Science, 144: 862-863.

4. Liu, S. X., Wang, C. L., Miao, Y. U., Li, Y. X. and Wang, E. B. 2005. Synthesis and Antiinfluenza Virus Activity of Polyoxometalates Containing Amantadine. Acta Chima. Sinica., 63: 1069-1074.

5. Al-Wahaibi, L., Hassan, H., Abo-Kamar, A., Ghabbour, H. and El-Emam, A. 2017. AdamantaneIsothiourea Hybrid Derivatives: Synthesis, Characterization, In Vitro Antimicrobial, and In Vivo Hypoglycemic Activities. Molecules, 22(5): 710.

6. Al-Omar, M. A., Al-Abdullah, E. S., Shehata, I. A. Habib, E. E., Ibrahim, T. M. and El-Emam, A. A. 2010. Synthesis, Antimicrobial, and Anti-inflammatory Activities of Novel 5-(1-adamantyl)-4arylideneamino-3-mercapto-1, 2,4-triazoles and Related Derivatives. Molecules, 15(4): 25262550.

7. Kapitsa, I. G., Suslov, E.V., Teplov, G. V., Korchagina, D. V., Komarova, N. I., Volcho, K. P., Voronina, T. A., Shevela, A. I. and Salakhutdinov N.F. 2012. Synthesis and anxiolytic Activity of 2 -aminoadamantane derivatives containing monoterpene fragments. Pharm. Chem. J., Rus. Orig., 46(5): 263-265.

8. Nief, O. A., Salman, H. N. and Ahamed, L. S. 2017. Synthesis, Characterization, Biological Activity Studies of Schiff Bases and 1, 3-Oxazepine Derived from 1,1-Bis(4-aminophenyl)-4Phenyl Cyclohexane. Iraqi J. Sci., 58(4B): 1998-2011.

9. Vuković, N., Sukdolak, S., Solujić, S. and Nićiforović, N. 2010. Substituted imino and amino derivatives of 4-hydroxycoumarins as novel antioxidant, antibacterial and antifungal agents: Synthesis and in vitro assessments. Food Chem., 120: 1011-1018.

10. De Souza, A. O., Galetti, F. C. S., Silva, C. L., Bicalho, B., Parma, M. M, Fonseca, S. F., Marsaioli, A. J., Trindade, A. C. L. B., Freitas-Gil, R. P., Bezerra, F. S., Neto M. A. and de Oliveira M. C. F. 2007. Antimycobacterial and cytotoxicity activity of synthetic and natural Compounds. Quim. Nova., 30(7): 1563-1566.

11. Alrecabi, Z. G., Alfraiji, R. A. J. and Al-Majidi, S. M. H. 2017. Synthesis, Identification of Some New Derivatives of Oxazepine, Thiazinone and Hydroquinazoline and Evaluation of Antibacterial Activity. Iraqi J. Sci., 58(3C): 1565-1579.

12. Khitam, T. A. 2016. Synthesis, Identification and Evaluation The Biological Activity for Some New Heterocyclic Compounds Derived from Schiff Bases. J. Appl. Chem., 9(5): 1-11.

13. Pham, V. H., Vu, B. D., Cao, D. N., Nguyen, V. T., Phan, T. P. D., Nguyen, Q. H. and Phan, D. C. 2019. Synthesis and bioactivity screening of some novel $\mathrm{N}$-(adamantan-1yl)-1-aryl-methanimines. J. mil. Pharm. Med., 2: 88-94.

14. Shukkur, A. H., Ali, S. I. and Ahmed, D. S. 2020. Synthesis of Six and Seven-membered Heterocyclic Molecules Containing an Adamantyl Fragment and an X-ray Crystal Structure of $(E)$ $N$-(adamantan-1-yl)-1-(3-nitrophenyl)methanimine. Baghdad Sci. J., 17(1): 272-286.

15. Al-Wahaibi, L. H., Al-Shaalan, N. H., Ghabbour, H. A., Tiekink, E. R. T. and El-Emam, A. A. 2019. Crystal structure of 3,5-bis(trifluoromethyl)benzyl(Z)-N'-(adamantan-1-yl)-4phenylpiperazine-1-carbothioimidate. C30H33F6N3S. Z. Kristallogr. NCS, 234: 1009-1012.

16. Al-Ghulikah, H. A., Ghabbour, H. A., Tiekink, E. R. T. and El-Emam, A. A. 2019. Crystal structure of 4-bromobenzyl (Z)-N-(adamantan1-yl)morpholine-4-carbothio-imidate. C22H29BrN2OS. Z. kristallogr. NCS, 234: 1001-1003.

17. Zhong, Q. 2019. Crystal structure of (Z)-2-((adamantan-1-ylimino)methyl)-5-methoxyphenol. C18H23NO2. Z. kristallogr. NCS, 234(2): 313-314.

18. Al-Mutairi, A. A., Al-Alshaikh, M. A., Ghabbour, H. A. , Tiekink, E. R.T. and El-Emam, A. A. 2020. Crystal structure of 1-(adamantan-1-yl)-3-aminothiourea, C11H19N3S. Z. Kristallogr. NCS, Ahead of Print, 18 Jun. 
19. Al-Wahaibi, L. H., Ghabbour, H. A., Mostafa, G. A. E., Almutairi, M. S. and El-Emam, A. A. 2016. Crystal structure of 1-(adamantan-1-yl)-3-phenylthiourea, C17H22N2S. Z. Kristallogr. NCS, 231: 593-595.

20. Jin, X. -D., Xu, C., Liu, X. -C., Yin, X .-Y., Gang, Y. -C., Yang, Q. and Jin, Y. -H. 2013. Synthesis, characterization, and crystal structure of two zinc (II) complexes with a Schiff base derived from amantadine. J. Coord. Chem., 66(22): 3970-3978.

21. PANalytical. 2011. X'Pert Data Collector and X'Pert Highscore-Plus. PANalytical BV, Almelo, The Netherlands.

22. Favre, N. V. and Cerny, R. Fox. 2002. Free Objects for Crystallography. J. Appl. Cryst., 35: 734743.

23. Zlokazov, V. B. and Chernyshev, V. V. MRIA. 1992. A program for full profile analysis of powder multiphase neutron-diffraction time-of-flight (direct and Fourier) spectra. J. Appl. Cryst., 25: $447-451$.

24. Macrae, C. F., Edgington, P. R., McCabe, P., Pidcock, E., Shields, G. P., Taylor, R., Towler, M. and van de Streek, J. Mercury. 2006. Visualization and analysis of crystal structures. J. Appl. Cryst., 39: 453-457. 